\title{
Investigation of biofilm production and its association with genetic and phenotypic characteristics of OM (osteomyelitis) and non-OM orthopedic Staphylococcus aureus
}

Shengpeng Yu ${ }^{1,2}$, Bei Jiang ${ }^{1,3}$, Chao Jia ${ }^{1}$, Hongri Wu${ }^{1}$, Jie Shen ${ }^{1}$, Xiaomei Hu ${ }^{3 *}$ and Zhao Xie ${ }^{1 *}$

\begin{abstract}
Background: Staphylococcus aureus is a primary pathogen of orthopedic infections. By mediating antimicrobial resistance, $S$. aureus biofilm plays an important role in the recalcitrance of orthopedic infections, especially for the intractable osteomyelitis (OM). This study investigated the relationship between biofilm production and various genetic or phenotypic characteristics among orthopedic S. aureus strains.
\end{abstract}

Methods: A total of 137 orthopedic S. aureus isolates were enrolled and divided into OM and non-OM groups. Biofilm production was evaluated using the crystal violet assay. Genetic and phenotypic characteristics including MRSA identification, MLST and spa typing, carriage of virulence genes, drug resistance, and patients' inflammatory responses indicators were characterized. The relationship between biofilm production and above-mentioned features was respectively analyzed among all isolates and compared between OM and non-OM isolates.

Results: Biofilm production presented no significant difference between OM (including 9 MRSA isolates) and nonOM (including 21 MRSA isolates) strains. We found that ST88, t377 and ST630-MSSA-t377 strains produced very strong biofilms, while MLST types of ST15, ST25, ST398, ST5, ST59 and spa types of t002, t2325, t437 tended to produce weaker biofilms. Strains with the following profiles produced stronger biofilms: fib(+)-hlgv(+)-lukED(+)-sei(-)-sem(-)seo(-) for all isolates, sei(-)-sem(-)-seo(-) for OM isolates, and cna (+)-fib (+)-hlgv (+)-lukED (+)-seb(-)-sed(-) for non-OM isolates. In addition, not any single drug resistance was found to be related to biofilm production. We also observed that, among OM patients, strains with stronger biofilms caused weaker inflammatory responses.

Conclusion: Some genetic or phenotypic characteristics of orthopedic strains were associated with biofilm production, and this association could be different among OM and non-OM strains. The results are of great significance for better understanding, evaluating and managing different kinds of biofilm-associated orthopedic infections, and provide potential targets for biofilm clearance.

Keywords: Staphylococcus aureus, Biofilm, Orthopedic infection, Osteomyelitis

*Correspondence: huxiaomei@tmmu.edu.cn; xiezhao54981@163.com 1 Department of Orthopedics, Southwest Hospital, Army Medical University, Gaotanyan Main Street 30\#, District Shapingba, Chongqing, China

${ }^{3}$ Department of Microbiology, College of Basic Medical Sciences, Army Medical University, Gaotanyan Main Street 30\#, District Shapingba, Chongqing, China

Full list of author information is available at the end of the article

\section{Introduction}

Staphylococcus aureus is a common and versatile grampositive pathogen in orthopedic patients $[1,2]$. The notorious bacterium attracts more and more attention because of its ability of producing various virulence factors and the worsening situation of drug resistance [3]. Biofilm is an important tool of pathogenic bacteria, and

c) The Author(s) 2020. This article is licensed under a Creative Commons Attribution 4.0 International License, which permits use, sharing, adaptation, distribution and reproduction in any medium or format, as long as you give appropriate credit to the original author(s) and the source, provide a link to the Creative Commons licence, and indicate if changes were made. The images or other third party material in this article are included in the article's Creative Commons licence, unless indicated otherwise in a credit line to the material. If material is not included in the article's Creative Commons licence and your intended use is not permitted by statutory regulation or exceeds the permitted use, you will need to obtain permission directly from the copyright holder. To view a copy of this licence, visit http://creativeco mmons.org/licenses/by/4.0/. The Creative Commons Public Domain Dedication waiver (http://creativecommons.org/publicdomain/ zero/1.0/) applies to the data made available in this article, unless otherwise stated in a credit line to the data. 
composed of bacterial communities and the polymeric matrix produced by them $[4,5]$. Biofilm-associated infections are challenging for anti-infectious therapy, because the biofilm structure provides an ideal shelter for the bacteria to survive from antimicrobial killing and clearance of immune system [5, 6]. It was reported that biofilm bacteria could be 10 to 1000 times more resistant than the planktonic bacteria [7].

Staphylococcus aureus biofilm infections are very common in orthopedic infections $[2,5,8]$. It has been widely recognized that this situation not only makes the bacteria difficult to eradicate, but also makes the infections tend to deteriorate into chronic and recurrent courses $[6,8]$. Osteomyelitis $(\mathrm{OM})$ is one of the most serious orthopedic infectious diseases, bringing huge physical and economic burdens to the patients [9]. The problem of $S$. aureus biofilm becomes more intractable when a patient suffers from OM infection $[8,10]$. Although the contribution of biofilm to $S$. aureus pathogenicity has been widely studied, few studies focused on exploring the relationship between biofilm production and genetic or phenotypic characteristics of orthopedic $S$. aureus isolates, especially for $S$. aureus $\mathrm{OM}$ isolates.

In a previous study conducted by our team [9], we collected and characterized the $S$. aureus isolates obtained from the orthopedic center of our hospital over a 2-year period. In consideration that genetic and phenotypic characteristics of bacteria strains are closely related to the biological and physiological properties $[6,8,11-13]$, we hypothesized that some of these characteristics might be associated with biofilm formation, and the association might be different between $\mathrm{OM}$ and non-OM isolates. Therefore, interested isolates from the previous study were included and divided into $\mathrm{OM}$ and non-OM isolates, biofilm production was measured using the crystal violet assay. The relationship between biofilm production and various genetic and phenotypic features (including the molecular type, carriage of the specific virulence gene, drug resistance, and inflammatory responses of corresponding patients) of the bacterial strains were investigated. Results of the study will reveal the genetic and phenotypic features of the S. aureus OM and nonOM strains with different capacities of biofilm production, and provide potential indicators for strong biofilm producers to help clinicians get early attention to the difficult-to-treat infections.

\section{Methods}

\section{Bacterial isolates}

This study was conducted at the orthopedic center of Southwest Hospital (First Affiliated Hospital of Army Military Medical University), a tertiary hospital in southwest China. All the available orthopedic S. aureus infection strains (162 isolates) isolated from inpatients of our center admitted from September 2013 to September 2015 were obtained [9]. All isolates were identified as $S$. aureus by phenotypic methods (API staphy system, Biomerieux), and further confirmed by PCR of the $S$. aureus-specific gene $f e m B[9,14]$. For the same patient, only the first positive culture was enrolled (23 isolates were excluded). Two isolates were excluded because of the missing of basic information. Finally, a total of 137 S. aureus isolates were included in this study. Included strains were divided into OM group (isolating from the marrow or its adjacent tissues of $S$. aureus OM infection, 60 isolates) and non-OM group (isolating from contemporaneous inpatients that had never been diagnosed as $\mathrm{OM}$, specimen sources include wounds, tissues, joint fluid, pus and blood, 77 isolates) [9].

\section{Definitions}

MRSA (methicillin-resistant Staphylococcus aureus) was identified when the mecA gene was positive on the genome of a $S$. aureus isolate by PCR method. An isolate was considered multi-drug resistance (MDR) when it was resistant to three or more classes of non- $\beta$-lactam antimicrobials. A clonal complex (CC) was defined to contain at least two STs sharing any six of the seven alleles [14]. S. aureus $\mathrm{OM}$ was diagnosed based on clinical symptoms, microbiology, histopathology, laboratory studies, and imaging examinations [15].

\section{Biofilm production assay}

Biofilm production was measured using the crystal violet assay. TSB (tryptic soy broth) medium supplemented with $1 \%$ glucose and $2 \% \mathrm{NaCl}$ was used for cultivation of biofilm production [16, 17]. Overnight culture of each isolate was respectively diluted 1:100 into $200 \mu \mathrm{l}$ medium in a 96-well flat-bottom plate (Costar-3599, USA). After incubation at $37{ }^{\circ} \mathrm{C}$ for $24 \mathrm{~h}$, the supernatants were removed, and the wells were washed three times with PBS. Biofilm was fixed in an incubator at $60^{\circ} \mathrm{C}$ for $15 \mathrm{~min}$, and then $150 \mu \mathrm{l}$ of $0.1 \%$ crystal violet solution was added to each well containing dry biofilm. After 15 min of staining, the plate was rinsed with PBS and air dried. Subsequently, $150 \mu \mathrm{l}$ of $33 \%$ acetic acid was added to each well to resuspend the stained biofilm, and the optical density at $492 \mathrm{~nm}\left(\mathrm{OD}_{492}\right)$ was measured using a Multiskan GO microplate reader (Thermo Scientific, USA). The well containing only sterile medium without bacteria inoculation was used as a negative control. A good biofilm former S. aureus ATCC 29213 was used as a positive control. Each isolate was tested for at least three biological repeats.

The production of biofilm formation was assessed by the $\mathrm{OD}_{492}$ value and multiples of cut-off OD value (ODc). 
ODc was calculated from arithmetic mean of the $\mathrm{OD}_{492}$ of negative controls with three times addition of standard deviation. Specifically, for each biological repeat, three negative control wells in each 96-well plate were designed (two 96-well plates were used in each biological repeat), the arithmetic mean of negative controls was then calculated. In this way, three arithmetic means could be acquired by three biological repeats, and then they were used for calculating the final arithmetic mean and standard deviation to get the ODc value. The following classification was applied to determine the capacity of biofilm formation: no biofilm production $(\mathrm{OD} \leq \mathrm{ODc})$, weak bioflim production (ODc $<\mathrm{OD} \leq 2 \mathrm{ODc}$ ), moderate biofilm production $(2 \mathrm{ODc}<\mathrm{OD} \leq 4 \mathrm{ODc})$, and strong biofilm production $(\mathrm{OD}>4 \mathrm{ODc})$ [18].

\section{Molecular genotyping and detection of virulence genes} Multiple molecular typing methods including MRSA identification, SCCmec typing of MRSA strains, MLST typing (to determine ST), and spa typing were performed as described in a previous study conducted by our team [9].

Thirty-six common virulence genes of $S$. aureus were detected by PCR amplification, including 11 adhesionassociated genes ( $b b p, c l f A, c l f B, c n a, e b p s$, eno, fib, fnbA, $f n b B$, icaA, and icaD), 12 enterotoxin genes (sea, seb, sec, sed, see, seg, seh, sei, sej, sem, sen, and seo), and 13 other virulence genes ( $h l a, h l b, h l d, h l g, h l g v, l u k M, l u k E D, p v l$, psma, tst, eta, etb, and edin). All the PCR experiments had been finished in our previously published article [9].

\section{Antimicrobial susceptibility data}

Antimicrobial susceptibility results containing 15 antimicrobials were acquired from the clinical laboratory database of Southwest Hospital. Tested antimicrobials included ciprofloxacin (CIP), clindamycin (CLI), erythromycin (ERY), gentamicin (GEN), linezolid (LNZ), levofloxacin (LVX), moxifloxacin (MFX), nitrofurantoin (NIT), oxacillin (OXA), penicillin (PEN), rifampicin (RIF), trimethoprim/sulfamethoxazole (SXT), tetracycline (TCY), tigecycline (TGC), and vancomycin (VAN). MIC criteria of Clinical and Laboratory Standards Institute (CLSI) were used to determine antimicrobial resistance.

\section{Other clinical and laboratory data}

Basic demographic data including age, sex were anonymously collected. Indicators usually used for evaluating the inflammatory responses of orthopedic patients, including peak values of C-reactive protein (CRP), erythrocyte sedimentation rate (ESR), white blood cell count (WBC), and absolute neutrophil count (ANC) values [9, 15], were collected from all the enrolled patients before an operation intervention and near the bacterial sampling time (because of non-specifically and extremely high values, CRP values of patients with basic inflammatory diseases or recent trauma history were excluded).

\section{Statistical analysis}

IBM SPSS Statistics 23.0 (IBM, Chicago, IL, USA) was used for statistical analysis. In majority of previous studies, categorical variables representing different levels of biofilm-forming capacities were usually used $[19,20]$. But the biofilm production in the same level could be very different, so in this study, we used numerical variables $\left(\mathrm{OD}_{492}\right.$ values) for statistical analyses to improve the reliability of this study [21]. Mann-Whitney $U$ test or Kruskal-Wallis $H$ test was used when comparing the biofilm production between two groups or among three or more groups, respectively. Pearson correlation or spearman rank correlation test was used for correlation analysis. All statistical tests were two-sided and $\mathrm{P}<0.05$ was considered statistically significant.

\section{Results}

An overview of biofilm production for studied orthopedic isolates

First of all, to get an overview of biofilm production for all enrolled isolates, the biofilm-forming capacities of all strains were graded. As shown in Table 1, 137 orthopedic

Table 1 An overview of biofilm production of studied orthopedic strains

\begin{tabular}{|c|c|c|c|}
\hline Biofilm formation & Total $(n=137)$ & OM $(n=60)$ & Non-OM $(n=77)$ \\
\hline Weak (OD $\leq 2 \mathrm{ODC}), \mathrm{n}(\%)$ & $18(13.1)$ & $9(15.0)$ & $9(11.7)$ \\
\hline Moderate (2ODc < OD $\leq 4 \mathrm{ODc}), \mathrm{n}(\%)$ & $36(26.3)$ & $14(23.3)$ & $22(28.6)$ \\
\hline Strong $(4 \mathrm{ODc}<\mathrm{OD} \leq 8 \mathrm{ODc}), \mathrm{n}(\%)$ & $36(26.3)$ & $16(26.7)$ & $20(26.0)$ \\
\hline Strong (8ODc < OD $\leq 12 \mathrm{ODc}), \mathrm{n}(\%)$ & $9(6.6)$ & $3(5.0)$ & $6(7.8)$ \\
\hline Strong (12ODc< $\mathrm{OD} \leq 16 \mathrm{ODc}), \mathrm{n}(\%)$ & $9(6.6)$ & $6(10.0)$ & $3(3.9)$ \\
\hline Strong (OD > 16ODc), n (\%) & $29(21.2)$ & $12(20.0)$ & $17(22.1)$ \\
\hline OM vs. non-OM & $p^{a}=0.946$ & & \\
\hline
\end{tabular}

${ }^{a}$ By Mann-Whitney $U$ test, indicating no significant difference in biofilm formation between OM and non-OM isolates. ODc $=0.073$ 
S. aureus isolates all formed biofilms at various degrees. Among them, 13.1\% (18/137), 26.3\% (36/137) and 60.6\% $(83 / 137)$ strains presented weak, moderate and strong biofilm production, respectively. More than one-fifth of the strains showed very strong biofilm-forming capacity, with the $\mathrm{OD}_{492}$ value $>16 \mathrm{ODc}$ (1.175). In addition, no significant difference of biofilm production was found in any group between different genders, or among different sample sources and different ages (Additional file 1: Table S1). The distribution of $\mathrm{OD}_{492}$ values between $\mathrm{OM}$ and non-OM groups showed no significant difference either $(P=0.946$, Table 1$)$. These results provided the basis for analyzing the relationship between biofilm production and molecular types among all the orthopedic isolates.

\section{Relationship between biofilm production and MLST or spa type}

To determine whether some specific MLST or spa types could produce more biofilms, MLST and spa typing results of the 137 isolates were summarized in Additional file 2: Table S2. Totally, 34 MLST types and 54 spa types were detected (24 MLST types and 36 spa types in OM group, 26 MLST types and 35 spa types in nonOM group), indicating diverse sources of the orthopedic $S$. aureus infection strains. Biofilm production was compared among various genotypes, and significant differences were found among different MLST and spa types $(P<0.001$, Fig. 1a, b). Specifically, we found that ST88 strains produced significantly stronger biofilms than ST15 $(P=0.006)$, ST25 $(P=0.002)$, ST398 $(P=0.001)$, ST5 $(P=0.025)$ and ST59 $(P=0.024)$ strains (Fig. 1a). As for spa types, biofilms produced by $\mathrm{t} 377$ strains were significantly stronger than those of t002 $(P=0.004)$, t2325 $(P<0.001), \mathrm{t} 437(P=0.004)$, and other spa types $(P=0.004)$. In addition, t189 strains were more likely to produce stronger biofilms than $\mathrm{t} 2335$ strains $(P=0.025)$ (Fig. 1b).

Next, we wondered if the predominant STs $(n \geq 10)$ in this study showed some special features when producing biofilms. The biofilm production of predominant STs was summarized in Fig. 1c. We noticed that among ST630 strains, ST630-MSSA-t377 strains all presented $\mathrm{OD}_{490}$ values of more than $18 \times \mathrm{ODc}$, while others (ST630-MRSA-SCCmecI-t4549/t1376) produced much weaker biolfims (Fig. 1c). ST59-MRSA-SCCmecI
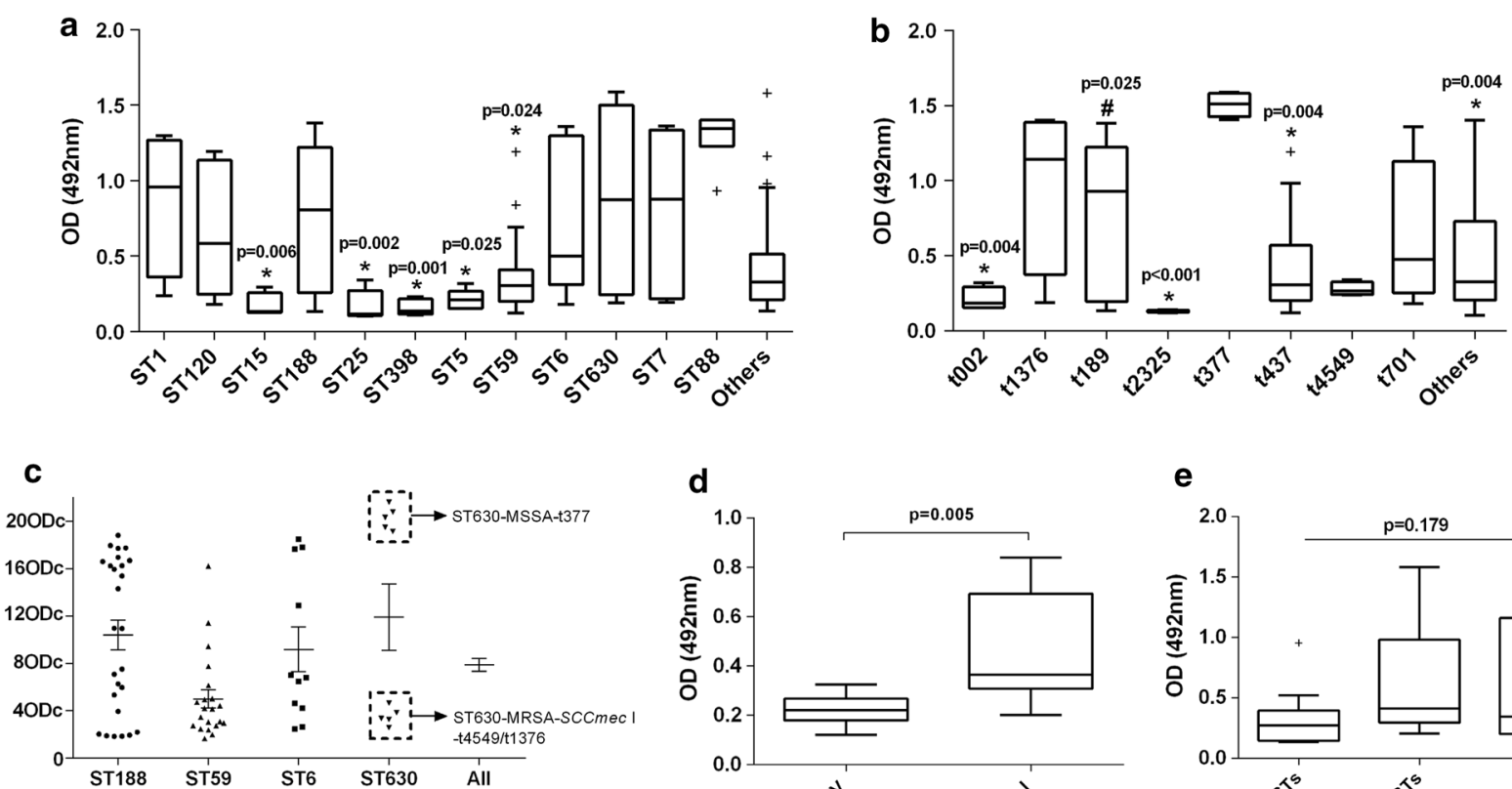

d

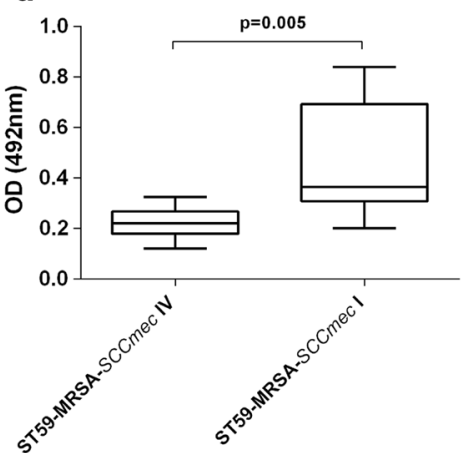

e

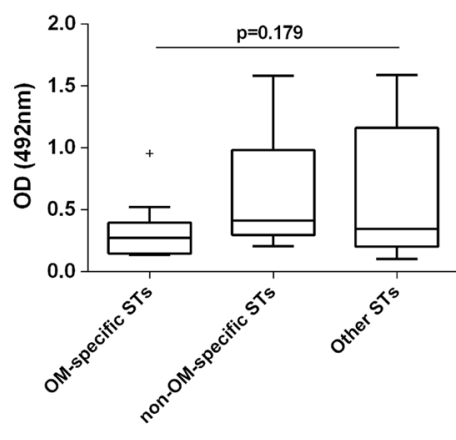

Fig. 1 Relationship between biofilm production and molecular genotypes. a Box and whisker plot showing the relationship between biofilm production and MLST types. $\mathbf{b}$ Box and whisker plot showing the relationship between biofilm production and spa types. $\mathrm{cOD}_{492}$ values (indicated by multiples of ODc) distributions of predominant STs $(n \geq 10)$. $\mathbf{d}$ Comparison of $\mathrm{OD}_{492}$ values between SCCmec I and SCCmec IV ST59-MRSA strains. e Comparison of $\mathrm{OD}_{492}$ values among OM-specific STs, non-OM-specific STs and other STs. "*" denotes statistically significant difference. "+" denotes outliers 
strains produced significantly stronger biofilms than ST59-MRSA-SCCmecIV strains $(P=0.005)$ (Fig. 1c, d). No clue was found to explain the different biofilm production among ST188 or ST6 strains. We also compared the biofilm formation among OM-specific STs, non-OMspecific STs and other STs, and no significant difference was found ( $P=0.179$, Fig. 1e).

\section{Relationship between biofilm production and carriage of the specific virulence gene}

In previous studies, it is controversial on the issue of whether virulence genes could be regarded as indicators for biofilm-forming capacity of $S$. aureus strains [11, 12, $19,22,23]$. To investigate this question among orthopedic $S$. aureus strains, the carriage of various virulence genes was tested and summarized in Table 2. The biofilm production was compared between specific gene-positive and gene-negative strains. Genes significantly associated with biofilm production were further subjected to spearman correlation analyses (Table 3 ).

For all enrolled strains, fib, hlgv and $l u k E D$ genes were positively correlated with biofilm production, while sei, sem and seo genes were negatively correlated. Interestingly, the biofilm production-associated genes were completely different for $\mathrm{OM}$ and non-OM strains. In $\mathrm{OM}$ group, no adhesion-associated gene was found to be correlated with biofilm production, and only three enterotoxin genes (sei, sem and seo) were found to be negatively correlated. Whereas in non-OM group, genes including $c n a, f i b, h l g v, l u k E D$ and eta were positively correlated with biofilm production, and another two enterotoxin genes, seb and sed, were negatively correlated with biofilm production (Tables 2 and 3 ).

Based on the above results, we further concluded that strains with the following virulence gene profiles presented significantly stronger biofilm production: 1) fib $(+)$-hlgv (+)-lukED (+)-sei (-)-sem (-)-seo (-) strains for all the orthopedic isolates $(P<0.001)$; 2$)$ sei $(-)$-sem $(-)$-seo $(-)$ strains for OM isolates $(P=0.006)$; 3$) c n a(+)-f i b(+)$ $h \operatorname{lgv}(+)-\operatorname{lukED}(+)$-seb(-)-sed (-) strains for non-OM isolates $(P<0.001)$ (Fig. 2).

\section{Relationship between biofilm production and drug resistance}

In clinical treatment of $S$. aureus-associated orthopedic infections, biofilm formation and drug-resistance make bacterial eradication more difficult, so we wonder if drug-resistance of $S$. aureus strains was associated with the biofilm formation ability [5, 19]. As summarized in Table 4, for all enrolled strains and non-OM strains, MRSA strains were found to produce significantly weaker biofilms. Weaker biofilms were also observed in the tetracycline-resistant strains due to a positive correlation
Table 2 Comparison of biofilm production between specific gene-positive and gene-negative strains in each group

\begin{tabular}{|c|c|c|c|}
\hline Strain trait & Total $(n=137)$ & OM $(n=60)$ & Non-OM $(n=77)$ \\
\hline \multicolumn{4}{|c|}{ Adhesion-associated genes, $\mathrm{n}(\%), P$ value $\mathrm{a}^{\mathrm{a}}$} \\
\hline$b b p(+)$ & $12(8.8), 0.670$ & $10(16.7), 0.330$ & $2(2.6), 0.259$ \\
\hline$c n a(+)$ & $47(34.3), 0.223$ & $21(35.0), 0.670$ & $26(33.8), 0.036$ \\
\hline ebps (+) & 40 (29.2), 0.558 & $24(40.0), 0.214$ & $16(20.8), 0.552$ \\
\hline$f i b(+)$ & $124(90.5), 0.006$ & $53(88.3), 0.120$ & $71(92.2), 0.014$ \\
\hline$f n b A(+)$ & $25(18.2), 0.737$ & $8(13.3), 0.433$ & $17(22.1), 0.810$ \\
\hline$f n b B(+)$ & $36(26.3), 0.401$ & $14(23.3), 0.832$ & $22(28.6), 0.182$ \\
\hline$i c a A(+)$ & $136(99.3)_{,}-$ & $59(98.3),-$ & $77(100.0),-$ \\
\hline \multicolumn{4}{|c|}{ Enterotoxin genes, $n(\%), P$ value ${ }^{a}$} \\
\hline sea $(+)$ & $11(8.0), 0.896$ & $7(11.7), 0.667$ & $4(5.2), 0.680$ \\
\hline $\operatorname{seb}(+)$ & $27(19.7), 0.234$ & $13(21.7), 0.634$ & $14(18.2), 0.032$ \\
\hline $\sec (+)$ & $16(11.7), 0.104$ & $8(13.3), 0.083$ & $8(10.4), 0.558$ \\
\hline $\operatorname{sed}(+)$ & $19(13.9), 0.052$ & $4(6.7), 0.785$ & $15(19.5), 0.015$ \\
\hline $\operatorname{see}(+)$ & $2(1.5), 0.892$ & $0(0),-$ & $2(2.6), 0.860$ \\
\hline $\operatorname{seg}(+)$ & $8(5.8), 0.677$ & $5(8.3), 0.566$ & $3(3.9), 0.159$ \\
\hline $\operatorname{seh}(+)$ & $4(2.9), 0.328$ & $0(0),-$ & $4(5.2), 0.342$ \\
\hline $\operatorname{sei}(+)$ & $26(19.0), 0.004$ & $14(23.3), 0.002$ & $12(15.6), 0.311$ \\
\hline $\operatorname{sej}(+)$ & $9(6.6), 0.237$ & $4(6.7), 0.657$ & $5(6.5), 0.250$ \\
\hline $\operatorname{sem}(+)$ & $29(21.2), 0.009$ & $15(25.0), 0.039$ & 14 (18.2), 0.112 \\
\hline $\operatorname{sen}(+)$ & $21(15.3), 0.066$ & $9(15.0), 0.356$ & $12(15.6), 0.121$ \\
\hline $\operatorname{seo}(+)$ & $32(23.4), 0.002$ & $19(31.7), 0.005$ & $13(16.9), 0.123$ \\
\hline \multicolumn{4}{|c|}{ Other toxin genes, $\mathrm{n}(\%), P$ value ${ }^{\mathrm{a}}$} \\
\hline hla $(+)$ & $132(96.4), 0.074$ & 55 (91.7), 0.073 & $77(100.0),-$ \\
\hline$h / b(+)$ & 45 (32.8), 0.194 & $18(30.0), 0.363$ & $27(35.1), 0.446$ \\
\hline$h \lg (+)$ & $127(92.7), 0.640$ & 58 (96.7), 0.916 & $69(89.6), 0.596$ \\
\hline$h \lg v(+)$ & $127(92.7), 0.005$ & 55 (91.7), 0.073 & $72(93.5), 0.035$ \\
\hline lukED (+) & $90(65.7)_{1}<0.001$ & $43(71.7), 0.364$ & $47(61.0),<0.001$ \\
\hline$p v l(+)$ & $26(19.0), 0.319$ & $17(28.3), 0.589$ & $9(11.7), 0.419$ \\
\hline edin $(+)$ & $8(5.8), 0.883$ & $4(6.7), 0.513$ & $4(5.2), 0.393$ \\
\hline eta $(+)$ & $5(3.6), 0.436$ & $2(3.3), 0.386$ & $3(3.9), 0.016$ \\
\hline tst $(+)$ & $5(3.6), 0.821$ & $3(5.0), 0.847$ & $2(2.6), 0.863$ \\
\hline
\end{tabular}

Significant differences are in italics

a By Mann-Whitney $\mathrm{U}$ test, comparing biofilm production ( $\mathrm{OD}_{492}$ values) between specific gene-positive and gene-negative strains in each group. Genes with carriage rate of $100 \%$ (eno, clfA, clfB,icaD, hld and psma) or $0 \%$ (lukM and etb) were not listed

between MRSA and tetracycline-resistant strains (Additional file 3: Table S3). No correlation was observed between biofilm production and any other kind of drug resistance in any group (Table 4 ).

\section{Relationship between biofilm production and patients' inflammatory responses}

It was reported in vitro and in the mouse model that bacterial biofilms could reduce the levels of inflammatory responses to keep the bacteria alive and simultaneously facilitate persistent infections [24, 25]. To explore 
Table 3 Spearman's correlation analyses between biofilm production and the carriage of specific virulence gene

\begin{tabular}{|c|c|c|c|}
\hline \multirow[t]{2}{*}{ Strain trait } & \multicolumn{3}{|c|}{ Spearman's rho and $P$ values } \\
\hline & Total & OM & Non-OM \\
\hline$c n a(+)$ & $P>0.05$ & $P>0.05$ & $r h o=0.250, P=0.028$ \\
\hline fib $(+)$ & rho $=0.234, P=0.006$ & $P>0.05$ & rho $=0.274, P=0.016$ \\
\hline $\operatorname{seb}(+)$ & $P>0.05$ & $P>0.05$ & rho $=-0.245, P=0.031$ \\
\hline $\operatorname{sed}(+)$ & $P>0.05$ & $P>0.05$ & rho $=-0.262, P=0.001$ \\
\hline $\operatorname{sei}(+)$ & $\mathrm{rho}=-0.246, P=0.004$ & rho $=-0.387, P=0.002$ & $P>0.05$ \\
\hline $\operatorname{sem}(+)$ & rho $=-0.224, P=0.008$ & $\mathrm{rho}=-0.268, P=0.039$ & $P>0.05$ \\
\hline seo $(+)$ & rho $=-0.263, P=0.002$ & $\mathrm{rho}=-0.360, P=0.005$ & $P>0.05$ \\
\hline$h \lg v(+)$ & rho $=0.235, P=0.006$ & $P>0.05$ & rho $=0.240, P=0.036$ \\
\hline lukED (+) & $\mathrm{rho}=0.283, P=0.001$ & $P>0.05$ & rho $=0.421, P<0.001$ \\
\hline eta $(+)$ & $P>0.05$ & $P>0.05$ & rho $=0.263, P=0.021$ \\
\hline
\end{tabular}
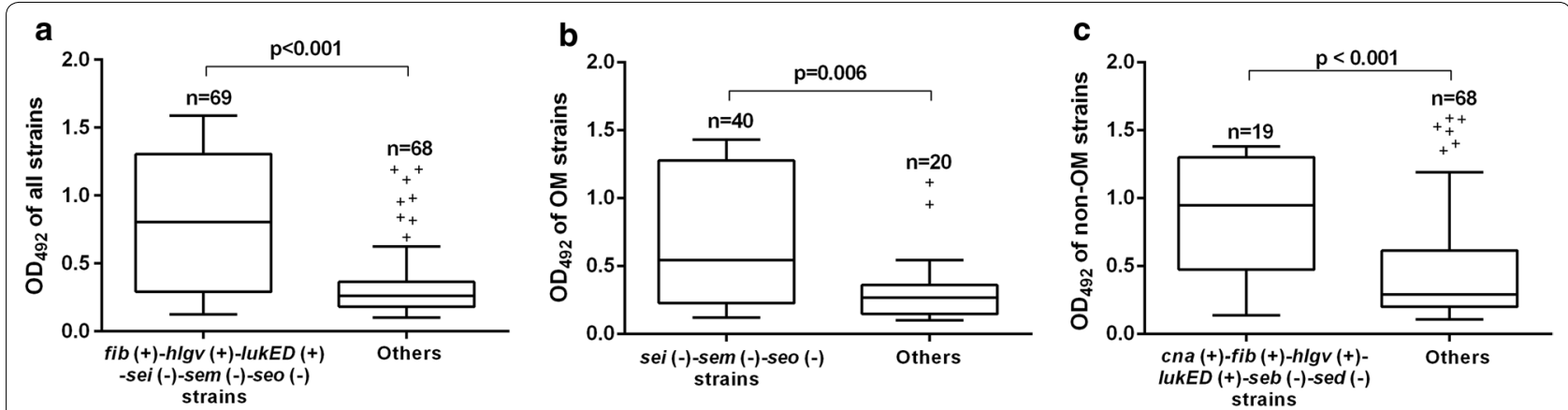

Fig. 2 Virulence gene profiles of strains with stronger biofilm production in each group (Box \& whisker plot). a For all studied orthopedic strains. b For OM strains. c For non-OM strains. "+" denotes outliers

Table 4 Relationship between biofilm production and drug resistance

\begin{tabular}{|c|c|c|c|}
\hline \multirow[t]{2}{*}{ Strain trait } & Total $(n=137)$ & $O M(n=60)$ & Non-OM $(n=77)$ \\
\hline & \multicolumn{3}{|l|}{$\mathrm{N}(\%), P$ value $^{\mathrm{a}}$} \\
\hline MRSA & $30(21.9), 0.038(\text { rho }=-0.169, P=0.048)^{b}$ & $9(15.0), 0.553$ & $\begin{array}{l}21(27.3), 0.042 \\
(r h o=-0.238 \\
P=0.037)^{b}\end{array}$ \\
\hline MDR & $40(29.2), 0.089$ & $18(30.0), 0.113$ & $22(28.6), 0.387$ \\
\hline CIP-resistant & $12(8.8), 0.533$ & $6(10.0), 0.641$ & $6(7.8), 0.191$ \\
\hline CLI-resistant & $62(45.3), 0.151$ & $27(45.0), 0.202$ & $35(45.5), 0.417$ \\
\hline ERY-resistant & $67(48.9), 0.245$ & $27(45.0), 0.390$ & $40(51.9), 0.454$ \\
\hline GEN-resistant & $12(8.8), 0.455$ & $6(10.0), 0.711$ & $6(7.8), 0.133$ \\
\hline LVX-resistant & $10(7.3), 0.260$ & $4(6.7), 0.598$ & $6(7.8), 0.368$ \\
\hline MFX-resistant & $9(6.6), 0.528$ & $4(6.7), 0.876$ & $5(6.5), 0.416$ \\
\hline OXA-resistant & $32(23.4), 0.456$ & $13(21.7), 0.395$ & $19(24.7), 0.776$ \\
\hline PEN-resistant & $125(91.2), 0.888$ & $53(88.3), 0.340$ & $72(93.5), 0.367$ \\
\hline RIF-resistant & $7(5.1), 0.121$ & $5(8.3), 0.256$ & $2(2.6), 0.836$ \\
\hline SXT-resistant & $13(9.5), 0.946$ & $4(6.7), 0.109$ & 9 (11.7), 0.318 \\
\hline TCY-resistant & $38(27.7), 0.031(r h o=-0.185, P=0.031)^{b}$ & $21(35.0), 0.098$ & $17(22.1), 0.133$ \\
\hline
\end{tabular}

All the strains were susceptible to LNZ, TGC and VAN. Significant differences are in italics

a By Mann-Whitney $\mathrm{U}$ test, comparing biofilm production $\left(\mathrm{OD}_{492}\right.$ values) between resistant and non-resistant strains in each group

b Significant factors were further subjected to spearman correlation analyses 
if this would happen to clinical orthopedic patients, we analyzed the relationship between levels of inflammatory response indicators (including CRP, ESR, WBC and $\mathrm{ANC}$ ) and the biofilm production of corresponding strains. As shown in Table 5, only for OM patients, CRP and ESR values were negatively correlated with the biofilm production of corresponding S. aureus strains. No correlation was detected between biofilm production and $\mathrm{WBC}$ or $\mathrm{ANC}$ value in any group.

\section{Discussion}

Drug resistance is a great concern in the treatment of infectious diseases [3], and biofilm plays an important role in causing drug resistance $[8,10,20]$. S. aureus is usually the most common pathogen in orthopedic infections, and skilled in biofilm formation [2, 8]. Hence, investigating the relationship between genetic or phenotypic characteristics and biofilm production among orthopedic $S$. aureus strains, especially for OM-infection strains, is of great significance for clinicians to understand, evaluate and manage biofilm-associated orthopedic infections.

Until now, the issue of whether the molecular type of $S$. aureus is associated with biofilm production is still controversial and lack of investigation. To our knowledge, this study is the first one that investigated the relationship between biofilm-forming capacities and molecular types among orthopedic S. aureus strains. According to the results from other specimen resources, some researchers reported that different spa types, but not MLST types, could present different biofilm production capacities [20, 26, 27]. However, in several other studies, MLST types were also found to be associated with biofilm-forming capacities. For example, Croes $\mathrm{S}$ et al. and Luther MK et al. suggested that MLST CC8 S. aureus produced more biofilms than various other MLST types $[20,28]$. In this study, we first proposed that ST88, t377 and ST630-MSSA-t377 strains were associated with very strong biofilm producers (Fig. 1a-c). ST88 S. aureus is a relatively common clone in Africa, but only sporadic infections were reported in China [29]. No research focusing on biofilm-forming capacity of ST88 S. aureus was found. However, it should be noted that a considerable proportion of ST88 S. aureus strains carried $p v l$ gene [29], a pore-forming toxin gene that plays a crucial role in the pathogenicity of S. aureus [9]. Five of the six $\mathrm{t} 377$ strains in this study belonged to ST630MSSA, ST630-MSSA-t377 strains presented as sporadic infections or relatively dominant strains in China [30, 31 , but the strong biofilm-forming capacity had not been realized in previous studies. Our results suggest that special attention should be paid to the biofilm infections caused by ST88 and ST630-MSSA-t377 strains, and larger epidemiological investigations are needed to verify their very strong biofilm-forming capacity and to explore the underlying mechanisms. For ST59, one of the most prevalent MRSA clones in Asia, although Yang X et al. reported that ST59-SCCmecIV strains isolated from Chinese children were more likely to form strong biofilm [21], our results showed that ST59-MRSA-SCCmecI produced significantly stronger biofilms than ST59-MRSASCCmecIV strains (Fig. 1d), suggesting that different environments or specimen sources might also affect the biofilm-forming capacity.

Exploring the relationship between biofilm production and carriage of virulence genes may provide biomarkers for diagnosis and targets for biofilm eradication. Although Tang $J$ et al. indicated that a single gene or subset of genes cannot be utilized as an indicator of biofilm production [23], In some other studies, traditional adhesion-associated genes including ebps, ica and $f n b A$ were suggested to be positively associated with biofilm production [11, 22]. Exotoxin gene lip and hla genes were also verified to contribute to biofilm formation [12, 32]. Different from the above results, in this study, we first found that the $f i b$, hlgv and lukED gene was positively correlated, while sei, sem and seo genes were negatively correlated with biofilm production of orthopedic $S$. aureus strains (Tables 2 , and 3 ). Interestingly, we noticed that genes correlated with biofilm production were completely different for OM and non-OM strains (Table 3). This result implies that different factors or mechanisms participate in the biofilm production of OM and nonOM strains. Also worth noting is that, in each group, we proposed a virulence gene profile with which the strains tended to produce stronger biofilm (Fig. 2). These

Table 5 Pearson's correlation analyses between biofilm formation and laboratory data

\begin{tabular}{llll}
\hline & Total (mean \pm SD, rho, $\boldsymbol{P})^{\mathbf{a}}$ & OM (mean \pm SD, rho, $\boldsymbol{P})^{\mathbf{a}}$ & Non-OM $(\mathbf{m e a n} \pm \text { SD, rho, } \boldsymbol{P})^{\mathbf{a}}$ \\
\hline CRP value & $49.4 \pm 80.2(n=109),-0.098,0.312$ & $34.6 \pm 71.8(n=52),-0.294,0.034$ \\
ESR value & $38.9 \pm 31.7(n=110),-0.113,0.240$ & $30.2 \pm 29.5(n=54),-0.287,0.035$ \\
WBC value & $8.6 \pm 4.3(n=118),-0.031,0.743$ & $8.8 \pm 4.7(n=57),-0.041,0.764$ \\
ANC value & $6.3 \pm 4.2(n=118), 0.002,0.987$ & $6.3 \pm 4.6(n=57),-0.013,0.926$ & $47.2 \pm 31.6(n=56), 0.098,0.475$ \\
\hline
\end{tabular}

Significant differences are in italics

a Pearson's correlation analyses between laboratory data and biofilm formation $\left(\mathrm{OD}_{492}\right)$ 
profiles probably reflect some features of strong biofilm producers.

It has been implied in many kinds of bacteria that antimicrobial susceptibility could be related to biofilm production [33-35]. But the exact relationship between them is still confusing. For $S$. aurues, several studies suggested that biofilm-forming strains were more likely to be MDR, and MRSA produced stronger biofilm than MSSA strains $[13,21]$. However, in some other studies, no correlation between drug resistance and biofilm-forming capacity was found [36]. In this study, only a negative correlation was found between MRSA and biofilm production among non-OM strains (Table 3). So we speculate that a single antimicrobial resistance is unable to alter the biofilm-forming capacity, but acquisition of the $m e c A$ gene, which mediates the transition from MSSA to MRSA [14], may change the process of biofilm-forming of orthopedic non-OM strains by unknown mechanisms.

To realize persistent infections (such as persistent $\mathrm{OM})$, bacteria usually need to adjust themselves to a state of low-level inflammatory responses, and biofilm formation is an important way to achieve this [24]. Although this phenomenon has been observed in vitro and in the mouse model [24, 25], and Klingenberg $C$ et al. also found that there was an association between a lower CRP value and biofilm-positive isolates in coagulase-negative staphylococci [36], it has not been verified in clinical patients with $S$. aureus orthopedic infections. Here, we found that, among OM patients, CRP and ESR values were negatively correlated with biofilm production (Table 5). This result further implies that, by reducing CRP and ESR values, bioflim plays a significant role in the course of persistent $\mathrm{OM}$ infections, and the two data could be potential indicators for biofilm-associated S. aureus OM infections.

\section{Conclusions}

In summary, we characterized the relationship between biofilm production and various genetic and phenotypic characteristics of orthopedic S. aureus strains isolated from OM and non-OM infections. Some MLST and spa types were shown to be associated with biofilm production. Strains with specific virulence gene profiles could be more likely to be strong biofilm producers, and this profile was completely different for $\mathrm{OM}$ and non-OM isolates. Not any single drug resistance was found to be associated with biofilm production. At last, we observed among OM patients that stronger biofilm producers tended to lead to lower inflammatory responses. The results of this study may help clinicians to better understand, evaluate and manage biofilm-associated orthopedic infections and provide potential targets for biofilm clearance.

\section{Supplementary information}

Supplementary information accompanies this paper at https://doi. org/10.1186/s12941-020-00352-4.

Additional file 1: Table S1. $\mathrm{OD}_{492}$ distribution between different genders, or among different sample sources, different ages in each group.

Additional file 2: Table S2. Molecular typing results of each group.

Additional file 3: Table S3. Correlation analyses between MRSA and TCYresistance by Kendall's tau test.

\section{Acknowledgements}

Not applicable.

\section{Authors' contributions}

$\mathrm{ZX}, \mathrm{XH}$ and BJ conceived and designed this study. SY, HW, JS and CJ carried out the experiments. BJ, SY, XH analyzed the data. BJ, ZX and XH drafted the manuscript. All authors read and approved the final manuscript.

\section{Funding}

This work was supported by Key Project (BWS13C014) of the General Logistics Department of PLA, and the General Program of National Natural Foundation of China (81672160, 31570127).

\section{Availability of data and materials}

All the dataset of this article is available from the corresponding author if reasonably requested.

\section{Ethics approval and consent to participate}

This study was approved by the Committee of the First Affiliated Hospital of Army Military Medical University [Approval Number: 2014KY [8]]. All the patients were anonymous and any other personal information was not used in this study.

\section{Consent for publication \\ Not applicable.}

\section{Competing interests}

All contributing authors declare no conflicts of interests.

\section{Author details}

${ }^{1}$ Department of Orthopedics, Southwest Hospital, Army Medical University, Gaotanyan Main Street 30\#, District Shapingba, Chongqing, China. ${ }^{2}$ Department of Orthopedics, Dujiangyan Medical Center, Dujiangyan, Sichuan, China. ${ }^{3}$ Department of Microbiology, College of Basic Medical Sciences, Army Medical University, Gaotanyan Main Street 30\#, District Shapingba, Chongqing, China.

Received: 2 September 2019 Accepted: 13 March 2020

Published online: 26 March 2020

\section{References}

1. Nishitani K, Sutipornpalangkul W, de Mesy Bentley KL, Varrone JJ, BelloIrizarry SN, Ito H, et al. Quantifying the natural history of biofilm formation in vivo during the establishment of chronic implant-associated Staphylococcus aureus osteomyelitis in mice to identify critical pathogen and host factors. J Orthop Res. 2015;33(9):1311-9.

2. Schwarz EM, Parvizi J, Gehrke T, Aiyer A, Battenberg A, Brown SA, et al. 2018 International consensus meeting on musculoskeletal infection: research priorities from the general assembly questions. J Orthop Res. 2019;37(5):997-1006.

3. David MZ, Daum RS. Treatment of Staphylococcus aureus infections. Curr Top Microbiol Immunol. 2017;409:325-83.

4. Del Pozo JL. Biofilm-related disease. Expert Rev Anti-infective Ther. 2018;16(1):51-65 
5. Saeed K, McLaren AC, Schwarz EM, Antoci V, Arnold WV, Chen AF, et al. 2018 International consensus meeting on musculoskeletal infection: summary from the biofilm workgroup and consensus on biofilm related musculoskeletal infections. J Orthop Res. 2019;37(5):1007-17.

6. Kumar A, Alam A, Rani M, Ehtesham NZ, Hasnain SE. Biofilms: survival and defense strategy for pathogens. Int J Med Microbiol. 2017;307(8):481-9.

7. Olson ME, Ceri H, Morck DW, Buret AG, Read RR. Biofilm bacteria: formation and comparative susceptibility to antibiotics. Can J Vet Res. 2002:66(2):86-92.

8. Zimmerli W, Sendi P. Orthopaedic biofilm infections. APMIS 2017;125(4):353-64.

9. Jiang $B$, Wang Y, Feng $Z$, Xu L, Tan L, Zhao S, et al. Panton-Valentine Leucocidin (PVL) as a potential indicator for prevalence, duration, and severity of Staphylococcus aureus Osteomyelitis. Front Microbiol. 2017;8:2355.

10. Brady RA, Leid JG, Calhoun JH, Costerton JW, Shirtliff ME. Osteomyelitis and the role of biofilms in chronic infection. FEMS Immunol Med Microbiol. 2008;52(1):13-22.

11. Bimanand L, Taherikalani M, Jalilian FA, Sadeghifard N, Ghafourian S, Mahdavi Z, et al. Association between biofilm production, adhesion genes and drugs resistance in different $\mathrm{SCCmec}$ types of methicillin resistant Staphylococcus aureus strains isolated from several major hospitals of Iran. Iranian J Basic Med Sci. 2018;21(4):400-3.

12. Anderson MJ, Schaaf E, Breshears LM, Wallis HW, Johnson JR, Tkaczyk C, et al. Alpha-toxin contributes to biofilm formation among Staphylococcus aureus wound isolates. Toxins. 2018;10(4):157.

13. Cha JO, Yoo Jl, Yoo JS, Chung HS, Park SH, Kim HS, et al. Investigation of biofilm formation and its association with the molecular and clinical characteristics of methicillin-resistant Staphylococcus aureus. Osong Public Health Res Perspect. 2013;4(5):225-32.

14. Cheng H, Yuan W, Zeng F, Hu Q, Shang W, Tang D, et al. Molecular and phenotypic evidence for the spread of three major methicillin-resistant Staphylococcus aureus clones associated with two characteristic antimicrobial resistance profiles in China. J Antimicrob Chemother. 2013;68(11):2453-7.

15. Lew DP, Waldvogel FA. Osteomyelitis. Lancet. 2004;364(9431):369-79.

16. Wu S, Yang T, Luo Y, Li X, Zhang X, Tang J, et al. Efficacy of the novel oxazolidinone compound FYL-67 for preventing biofilm formation by Staphylococcus aureus. J Antimicrob Chemother. 2014;69(11):3011-9.

17. Liu H, Shang W, Hu Z, Zheng Y, Yuan J, Hu Q, et al. A novel SigB(Q225P) mutation in Staphylococcus aureus retains virulence but promotes biofilm formation. Emerg Microbes Infect. 2018;7(1):72.

18. Xu Z, Liang Y, Lin S, Chen D, Li B, Li L, et al. Crystal violet and XTT assays on Staphylococcus aureus biofilm quantification. Curr Microbiol. 2016;73(4):474-82.

19. Ohadian Moghadam S, Pourmand MR, Aminharati F. Biofilm formation and antimicrobial resistance in methicillin-resistant Staphylococcus aureus isolated from burn patients, Iran. J Infect Dev Countries. 2014;8(12):1511-7.

20. Luther MK, Parente DM, Caffrey AR, Daffinee KE, Lopes VV, Martin ET, et al. Clinical and genetic risk factors for biofilm-forming Staphylococcus aureus. Antimicrob Agents Chemother. 2018;62(5):e02252-17.

21. Yang X, Qian S, Yao K, Wang L, Liu Y, Dong F, et al. Multiresistant ST59SCCmec IV-t437 clone with strong biofilm-forming capacity was identified predominantly in MRSA isolated from Chinese children. BMC Infect Dis. 2017:17(1):733.

22. Puah SM, Tan J, Chew CH, Chua KH. Diverse profiles of biofilm and adhesion genes in Staphylococcus Aureus food strains isolated from Sushi and Sashimi. J Food Sci. 2018;83(9):2337-42.
23. Tang J, Chen J, Li H, Zeng P, Li J. Characterization of adhesin genes, staphylococcal nuclease, hemolysis, and biofilm formation among Staphylococcus aureus strains isolated from different sources. Foodborne Pathog Dis. 2013;10(9):757-63.

24. Trouillet-Assant S, Lelievre L, Martins-Simoes P, Gonzaga L, Tasse J, Valour F, et al. Adaptive processes of Staphylococcus aureus isolates during the progression from acute to chronic bone and joint infections in patients. Cell Microbiol. 2016;18(10):1405-14.

25. Thurlow LR, Hanke ML, Fritz T, Angle A, Aldrich A, Williams SH, et al. Staphylococcus aureus biofilms prevent macrophage phagocytosis and attenuate inflammation in vivo. J Immunol (Baltimore, Md : 1950). 2011;186(11):6585-96.

26. Atshan SS, Shamsudin MN, Lung LT, Sekawi Z, Ghaznavi-Rad E, Pei CP. Comparative characterisation of genotypically different clones of MRSA in the production of biofilms. J Biomed Biotechnol. 2012;2012:417247.

27. Naicker PR, Karayem K, Hoek KG, Harvey J, Wasserman E. Biofilm formation in invasive Staphylococcus aureus isolates is associated with the clonal lineage. Microb Pathog. 2016;90:41-9.

28. Croes S, Deurenberg RH, Boumans ML, Beisser PS, Neef C, Stobberingh EE. Staphylococcus aureus biofilm formation at the physiologic glucose concentration depends on the S. aureus lineage. BMC Microbiol. 2009;9:229.

29. Moremi N, Claus H, Vogel U, Mshana SE. The role of patients and healthcare workers Staphylococcus aureus nasal colonization in occurrence of surgical site infection among patients admitted in two centers in Tanzania. Antimicrob Resist infection Control. 2019;8:102.

30. Yu F, LiT, Huang $X, X i e J, X u Y, T u J$, et al. Virulence gene profiling and molecular characterization of hospital-acquired Staphylococcus aureus isolates associated with bloodstream infection. Diagn Microbiol Infect Dis. 2012;74(4):363-8

31. Jiang W, Zhou Z, Zhang K, Yu Y. Epidemiological investigation of community-acquired Staphylococcus aureus infection. Genet Mol Res GMR. 2013;12(4):6923-30

32. Nguyen MT, Luqman A, Bitschar K, Hertlein T, Dick J, Ohlsen K, et al. Staphylococcal (phospho)lipases promote biofilm formation and host cell invasion. Int J Med Microbiol IJMM. 2018;308(6):653-63.

33. Vuotto C, Longo F, Pascolini C, Donelli G, Balice MP, Libori MF, et al. Biofilm formation and antibiotic resistance in Klebsiella pneumoniae urinary strains. J Appl Microbiol. 2017;123(4):1003-18.

34. Han X, Li Q, Shen L, Hu D, Qu Y. Correlation between the biofilm-forming ability, biofilm-related genes and antimicrobial resistance of Acinetobacter baumannii. Zhonghua wei zhong bing ji jiu yi xue. 2014;26(9):639-43.

35. Barakat GI, Nabil YM. Correlation of mupirocin resistance with biofilm production in methicillin-resistant Staphylococcus aureus from surgical site infections in a tertiary centre, Egypt. J Global Antimicrobial Resist. 2016:4:16-20

36. Klingenberg C, Aarag E, Ronnestad A, Sollid JE, Abrahamsen TG, Kjeldsen $\mathrm{G}$, et al. Coagulase-negative staphylococcal sepsis in neonates. Association between antibiotic resistance, biofilm formation and the host inflammatory response. Pediatr Infect Dis J. 2005;24(9):817-22.

\section{Publisher's Note}

Springer Nature remains neutral with regard to jurisdictional claims in published maps and institutional affiliations.

Ready to submit your research? Choose BMC and benefit from:

- fast, convenient online submission

- thorough peer review by experienced researchers in your field

- rapid publication on acceptance

- support for research data, including large and complex data types

- gold Open Access which fosters wider collaboration and increased citations

- maximum visibility for your research: over $100 \mathrm{M}$ website views per year

At BMC, research is always in progress.

Learn more biomedcentral.com/submissions 